\title{
Jugendliche und Polizei -Eine vergleichende Untersuchung zur Rolle verdachtsunabhängiger Personenkontrollen in französischen und deutschen Städten
}

\section{Dietrich Oberwittler}

Max-Planck-Institut für ausländisches und internationales Strafrecht, Abt. Kriminologie, Freiburg

erscheint in:

Recht der Jugend und des Bildungswesens 64(4), 2016, S. 414-427

ISSN (Print) 0034-1312

\section{d.oberwittler@mpicc.de}




\section{Jugendliche und Polizei Eine vergleichende Untersuchung zur Rolle verdachtsunabhängiger Personenkontrollen in französischen und deutschen Städten}

\section{Einleitung}

Die Ausbildung sozialer Identitäten ist ein wichtiger Teil der Sozialisation und stellt Jugendliche mit Migrationshintergrund vor besondere Herausforderungen, sind sie doch bei der Entwicklung eines nationalen Zugehörigkeitsgefühls den Einflüssen und Ansprüchen sowohl des Herkunfts- als auch des Aufnahmelandes ausgesetzt. In den europäischen Einwanderungsgesellschaften entstehen dabei häufig duale oder hybride Identitäten, in denen sich diese unterschiedlichen Einflüsse mischen. ${ }^{1}$ In den letzten Jahren hat dieses Thema durch den islamistischen Extremismus und Terror besondere Brisanz erfahren. Jugendliche aus muslimischen Herkunftsländern sind für islamistischen Extremismus dann besonders anfällig, wenn ihre soziale Identitätsbildung „schiefgelaufen“ ist und sie sich ihrer Aufnahmegesellschaft nicht nur nicht zugehörig fühlen, sondern sogar eine explizit feindselige Haltung zu ihr entwickeln. ${ }^{2}$

Steht heute der islamistische Terrorismus im Mittelpunkt der Aufmerksamkeit, so waren es zuvor gewaltsame Ausschreitungen von Jugendlichen in sozial benachteiligten Großstadtvierteln in Frankreich, Großbritannien und auch Schweden. Auch für diese sich spontan entladende Form der Gewalt werden soziale Frustrationen und Hass gegen die Aufnahmegesellschaft als zugrunde liegende Ursachen verantwortlich gemacht. Dieser Hass richtete sich bei den gewaltsamen Protesten gegen die Polizei, und zwar nicht nur deswegen, weil sie meistens durch Polizeieinsätze, bei denen migrantische Jugendliche zu Schaden kamen, ausgelöst wurden, ${ }^{3}$ sondern auch, weil Polizisten wichtige Repräsentanten des Staates sind. Neben der Schule stellt die Polizei vermutlich diejenige staatliche Institution dar, mit der Jugendliche im Alltagsleben am häufigsten konkrete Erfahrungen sammeln. Daher ist die Frage von anhaltender Bedeutung, wie diese Erfahrungen beschaffen sind, und inwiefern das Verhältnis zwischen

\footnotetext{
${ }^{1}$ Verkuyten, M./Fleischmann, F., Ethnicity, religion and nationality: Group identifications among minority youth, in: Rutland, A./Nesdale, D./Spears Brown, C. (Hrsg.), Handbook of group processes in children and adolescents, Oxford 2016.

${ }^{2}$ Maxwell, R./Bleich, E., What Makes Muslims Feel French? Social Forces 93 (1), 2014, S. 155-179

3 Waddington D., Applying the Flashpoints Model of Public Disorder to the 2001 Bradford Riot, British Journal of Criminology 50, 2010, S. 342-359.
} 
Jugendlichen und der Polizei einen positiven oder negativen Beitrag zur Identifikation von migrantischen Jugendlichen mit der Aufnahmegesellschaft leistet. Dabei ist klar, dass die (mangelnde) gesellschaftliche Integration von migrantischen Jugendlichen das Ergebnis sehr komplexer sozialer Prozesse ist, deren Komponenten nicht wirklich isoliert betrachtet werden können. ${ }^{4}$

414

Dieser Aufsatz beleuchtet diese Fragestellung anhand der Ergebnisse eines empirischen Forschungsprojekts zu den Erfahrungen von Jugendlichen mit der Polizei in Deutschland und Frankreich. Im Rahmen des Projekts POLIS - „Police and Adolescents in Multi-Ethnic Societies“ - wurden in zwei deutschen und zwei französischen Großstädten eine standardisierte Schulbefragung von insgesamt über 20.000 Jugendlichen sowie umfangreiche teilnehmende Beobachtungen und Interviews mit Polizisten durchgeführt. ${ }^{5}$ Im Mittelpunkt der folgenden Auswertungen steht die Frage, in welchem Ausmaß Jugendliche unterschiedlicher ethnischer Herkunftsgruppen verdachtsunabhängigen Personenkontrollen der Polizei ausgesetzt sind, welche Erfahrungen sie dabei machen, und in welcher Beziehung dies zu ihrem Vertrauen in die Polizei steht. Führen Personenkontrollen und insbesondere ihre unfaire und diskriminierende Anwendung zu einem Vertrauensverlust der Polizei bei Jugendlichen? Das Projekt vergleicht dabei die Erfahrungen zweier europäischer Nachbarländer, die sich sowohl hinsichtlich kollektiver Jugendgewalt und religiös motivierter Gewalt als auch hinsichtlich des Verhältnisses von Jugend und Polizei deutlich unterscheiden. Das POLIS-Projekt kann den bisherigen Kenntnisstand, der auf nur wenigen deutsch-französischen Vergleichsstudien basiert, mit seinen umfangreichen quantitativen und qualitativen Datenerhebungen deutlich erweitern. ${ }^{6}$

\section{Verdachtsunabhängige Personenkontrollen - Einfallstor für eine diskriminierende und unfaire}

\section{Behandlung durch die Polizei?}

${ }^{4}$ Alba, R. /Foner, N., Strangers No More: Immigration and the Challenges of Integration in North America and Western Europe, Princeton 2015; Loch, D., Pourquoi n'y a-t-il pas d'émeutes urbaines en Allemagne? Les jeunes issus de l'immigrations en France et en Allemange entre (absence de) protestations et politiques de la ville, Swiss Journal of Sociology, 2008, S. 281-306; Tucci I./Groh-Samberg O., Das enttäuschte Versprechen der Integration:

Migrantennachkommen in Frankreich und Deutschland, Schweizerische Zeitschrift für Soziologie 34, 2008, S. 307-333.

${ }^{5}$ Das Projekt wurde im Rahmen des ANR-DFG-Förderprogramms für die Geistes- und Sozialwissenschaften, Ausschreibung 2008, gefördert (Al 376-11/1). Projektleiter in Frankreich waren Sebastian Roché und in Deutschland Hans-Jörg Albrecht und Dietrich Oberwittler.

${ }^{6}$ Gauthier, J., Origines controllées. La police à l'épreuve de la question minoritaire à Paris et à Berlin (Dissertation Université Versailles-Saint-Quentin-en-Yvelines/Albert-Ludwigs-Universität $\quad$ Freiburg), 2012; Keller, C./Gauthier, $J$., Kontrollpolitik und Konflikteskalation in benachteiligte Stadtteilen. Ein deutsch-französischer Vergleich, in: Soeffner, H.-G. (Hrsg.), Unsichere Zeiten. Herausforderungen gesellschaftlicher Transformationen (DVD), Frankfurt 2010. 
Die Autoren einer der ersten Studien zu den gewaltsamen Unruhen in England im August 2011 fassten ihre Interviews mit Teilnehmern der Unruhen unter anderem so zusammen: „Ihre häufigste Beschwerde bezog sich auf die alltäglichen Erfahrungen mit der Polizei, und viele äußerten eine tiefsitzende Frustration über die Art und Weise, wie ihresgleichen Personenkontrollen unterworfen sind“" (Übersetzung D.O.).? Personenkontrollen, die nicht in Reaktion auf Straftaten oder sonstige Anlässe, sondern ohne konkreten Verdacht erfolgen, gehören zum Standardrepertoire polizeilicher Arbeit und stellen die vermutlich häufigste Art dar, mit der Polizei in Kontakt zu kommen. Zwar sind Personenkontrollen in Deutschland nach den Landespolizeigesetzen an einen orts- oder situationsbezogenen Verdacht gebunden, dass Straftaten vorbereitet werden oder sich dort Straftäter aufhalten, jedoch ist dieser Verdacht nicht notwendigerweise auf individuelle Personen gerichtet. Gerade bei Jugendlichen setzen Polizeibeamte dieses Mittel sehr großzügig und häufig auch ohne Verdachtsmomente ein, wie die empirischen Erhebungen des POLIS Projekts zeigen (siehe unten, insb. FN 22). Verdachtsunabhängige Personenkontrollen eröffnen der Polizei einen breiten Ermessensspielraum bei der Entscheidung, ob und wer kontrolliert wird. In vielen Ländern ist die Praxis der Personenkontrollen umstritten, weil dieser Ermessensspielraum potenziell zur Diskriminierung ethnischer Minderheiten führen kann. Dies gilt vor allem, wenn die Polizei ein sogenanntes „Ethnic Profiling“ praktiziert, also gezielt Personen aufgrund äußerer Merkmale, die auf die ethnische Zugehörigkeit hindeuten, kontrolliert, weil sie sich davon eine höhere „Erfolgsquote“ er-

hofft. In Großbritannien und den USA hat sich eine intensive Forschung zu der dort als „stop and search“ oder „stop and frisk“ genannten Polizeipraxis entwickelt. ${ }^{8}$ Da verdachtsunabhängige Personenkontrollen in Großbritannien registriert werden, ist bekannt, dass die Wahrscheinlichkeit einer Personenkontrolle in England \& Wales - bei einer Gesamtzahl von jährlich über einer Million Kontrollen für Schwarze etwa dreimal höher ist als für Weiße, ${ }^{9}$ und rechnerisch auf jeden 16-jährigen Jugendlichen in Glasgow und Umgebung 1,4 Personenkontrollen pro Jahr kommen. ${ }^{10}$

\footnotetext{
7 The Guardian, 5. Dezember 2011.

${ }^{8}$ Bradford, B./Loader, I., Police, crime and order: the case of stop and search, in: Bradford, B. u.a. (Hrsg.), The SAGE Handbook of Global Policing, London 2016, S. 241-260; Delsol, R./Shiner, M. (Hrsg.), Stop and Search. The Anatomy of a Police Power, London 2015; Fagan, J./Geller, A., Following the Script: Narratives of Suspicion in Terry Stops in Street Policing (Columbia Public Law \& Legal Theory Working Paper), 2014; Gelman A./Fagan J./Kiss, A., An Analysis of the New York City Police Department's "Stop-and-Frisk" Policy in the Context of Claims of Racial Bias, Journal of the American Statistical Association 102, 2007, S. 813-823.

${ }^{9}$ United Kingdom, Ministry of Justice, Statistics on Race and the Criminal Justice System 2012. A Ministry of Justice publication under Section 95 of the Criminal Justice Act 1991, London 2013; Borooah, V., Racial Disparity in Police Stop and Searches in England and Wales, Journal of Quantitative Criminology 27, 2011, S. 453-473.

${ }^{10}$ Murray, K., Stop and search in Scotland: An evaluation of police practice, Edinburgh 2014.
} 
Wie in den meisten anderen europäischen Ländern gibt es demgegenüber in Deutschland und Frankreich keine offiziellen Statistiken zu Personenkontrollen. Auf der Basis verdeckter Beobachtungen an Pariser Bahnhöfen ermittelten französische Forscher eine vielfach höhere Kontrollwahrscheinlichkeit Schwarzer gegenüber Weißen," und eine repräsentative Bevölkerungsbefragung im Auftrag der Agentur der Europäischen Union für Menschenrechte erbrachte sowohl für Frankreich als auch für Deutschland etwa doppelt so häufige Kontrollen von Angehörigen nordafrikanischer bzw. türkischer Minderheiten. ${ }^{12}$ Insgesamt bleibt der empirische Kenntnisstand jedoch sehr unbefriedigend und nicht ohne weiteres auf Jugendliche übertragbar. Empirische Studien zu den Erfahrungen Jugendlicher mit der Polizei in Deutschland haben kein einheitliches Bild erbracht. ${ }^{13}$ Dies gilt auch für die rechtliche Situation, nach der ethnische Diskriminierungen im Grundsatz unzulässig, aber in der Praxis schwer zu definieren und zu belegen sind..$^{14}$

Die kriminologische und sozialpsychologische Forschung hat unabhängig von der rechtlichen Situation ein relativ klares Bild entwickelt, warum diskriminierende Personenkontrollen einem positiven Verhältnis zwischen Bürgern und der Polizei abträglich sind. Hierfür sind die Begriffe Vertrauen und Legitimität zentral. In Anlehnung an Max Webers Herrschaftssoziologie hat der Sozialpsychologe Tom Tyler die Legitimität - das heißt die freiwillige Bereitschaft zur Anerkennung und Folgsamkeit - als wesentliche Voraussetzung einer erfolgreichen Polizeiarbeit be-

nannt. ${ }^{15}$ Die zentrale Annahme seiner Theorie der prozeduralen Gerechtigkeit lautet, dass die Legitimität von der Wahrnehmung der Betroffenen abhängt, gerecht, fair und respektvoll behandelt zu werden. Jede (auch indirekte) Erfahrung unfairer, respektloser oder gar brutaler Behandlung durch die Polizei führt demnach zu einem starken

${ }^{11}$ Goris, I./Jobard, F./Lévy, R., Profiling Minorities. A Study of Stop-and-Search Practices in Paris, New York 2009; Jobard, $F$./Lévy, R., Polizei, Justiz und rassistische Diskriminierungen in Frankreich, in: Fereidooni, M. (Hrsg.), Rassismuskritik und Widerstandsformen, Wiesbaden 2017, S. 529-544.

${ }^{12}$ European Union Agency for Fundamental Rights, EU-MIDIS European Union Minorities and Discrimination Survey. Data in Focus Report: Police Stops and Minorities, Brüssel 2010.

13 Gesemann, F., "Ist egal, ob man Ausländer ist oder so jeder Mensch braucht die Polizei." Die Polizei in der Wahrnehmung junger Migranten, in: Groenemeyer, A./Mansel, J. (Hrsg.), Die Ethnisierung von Alltagskonflikten, Opladen 2003 , S. 203- 228; Schweer, T./Strasser, H., "Die Polizei - Dein Freund und Helfer?!" Duisburger Polizisten im Konflikt mit ethnischen Minderheiten und sozialen Randgruppen, in: Groenemeyer, A./Mansel, J., Die Ethnisierung von Alltagskonflikten, 2003, S. 229-260.

14 Amnesty International, Racial/Ethnic Profiling: Positionspapier zu menschenrechtswidrigen Personenkontrollen, o.J. (Internet-Veröffentlichung, https://www.amnesty.de/files/Racial_Profiling_Positionspapier.pdf, abgerufen am 8.11.2016); Cremer, H., „Racial Profiling“ - Menschenrechtswidrige Personenkontrollen nach $\S 22$ Abs. 1a Bundespolizeigesetz. Empfehlungen an den Gesetzgeber, Gerichte und Polizei, Berlin 2013; Schicht G., Racial Profiling bei der Polizei in Deutschland - Bildungsbedarf? Beratungsresistenz?, Zeitschrift für internationale Bildungsforschung und Entwicklungspädagogik 36, 2013, S. 32-37.

15 Tyler, T., Why People Obey the Law, New Haven 1990; Tyler T., Trust and legitimacy: Policing in the USA and Europe, European Journal of Criminology 8, 2011, S. 254-266. 
Vertrauens verlust und erschwert in der Folge die Arbeit der Polizei: "The quickest way for the police to lose public consent is through mistreatment and unjust action." 16

Für Angehörige ethnischer Minderheiten ist die Wahrnehmung von Fairness und Respekt in besonderer Weise aufgeladen, denn individuelle Begegnungen mit der Polizei werden symbolisch auch als kollektive Begegnungen der Herkunftsgruppe mit der Mehrheitsgesellschaft und dem Staat wahrgenommen, und eine unfaire oder respektlose Behandlung durch Polizeibeamte kann dann als implizite Botschaft einer Geringschätzung ihrer sozialen Gruppe interpretiert werden. ${ }^{17}$ Diskriminierungen wie das Ethnic Profiling werden von den Betroffenen als ungerecht und stigmatisierend empfunden, weil eine Personenkontrolle nicht aufgrund des individuellen Verhaltens, sondern aufgrund von Gruppenmerkmalen wie der Hautfarbe erfolgt und damit dem Gleichheitsgrundsatz widerspricht. Nach Ansicht der Agentur der Europäischen Union für Menschenrechte sprechen nicht nur rechtliche, sondern auch polizeistrategische Gründe gegen diskriminierende Personenkontrollen, da sie die Legitimität der Polizei bei Migrantengruppen untergräbt. ${ }^{18}$ Gewaltsame Proteste gegen die Polizei können in diesem Sinne als extremes Beispiel für verlorenes Vertrauen gewertet werden. ${ }^{19}$

Diese Überlegungen bilden die Grundlage für die empirischen Analysen in diesem Beitrag. Hat die Polizei bei Jugendlichen aus ethnischen Minderheiten ein Vertrauensproblem, und steht dies möglicherweise mit diskriminierenden verdachtsunabhängigen Personenkontrollen im Zusammenhang? Lassen sich mit systematisch erhobenen Daten diejenigen Unterschiede bestätigen und ansatzweise auch erklären, die sich durch die vielfachen gewaltsamen Jugendproteste in Frankreich und ihr (bisheriges) Ausbleiben in Deutschland manifestiert haben?

\section{Datengrundlage}

Als ein Kernelement der POLIS-Studie fand 2011 und 2012 in den beiden deutschen Städten Köln und Mannheim sowie in den beiden französischen Städten Lyon und Grenoble eine standardisierte

${ }^{16}$ Jackson, J./Bradford, B./Stanko, E./Hohl, K., Just Authority? Trust in the Police in England and Wales, London/New York 2013, S. 7.

17 Tyler T./Blader, S., The Group Engagement Model: Procedural Justice, Social Identity, and Cooperative Behavior, Personality and Social Psychology Review 7, 2003, S. 349-361.

${ }^{18}$ Agentur der Europäischen Union für Grundrechte, Für eine effektivere Polizeiarbeit. Diskriminierendes „Ethnic Profiling“ erkennen und vermeiden: ein Handbuch, 2010.

19 Bradford, B./Jackson, J., Why Britons Trust their Police, 2011 (Internet-Veröffentlichung http://www.booksandideas.net/Why-Britons-Trust-their-Police.html, abgerufen am 8.11.2016). 
Schulbefragung mit weitgehend identischem Fragebogen und bedeutender Stichprobengröße statt $(\mathrm{ca} . \mathrm{n}=$ 7.300 in Deutschland, ca. $n=14.000$ in Frankreich). Die Anlage und Größe der Stichprobe ermöglicht verallgemeinerungsfähige Aussagen über Jugendliche in den beteiligten Städten in der Altersgruppe von ca. 13 bis 16 Jahren. Das Erhebungsinstrument enthielt Fragen $\mathrm{zu}$ dem soziodemografischen und familiären Hintergrund der Schüler, zu Schule und Freizeitverhalten, zum delinquenten Verhalten und schließlich auch zu ihrem Verhältnis zur Polizei. Ein

Bericht mit deskriptiven Ergebnissen der Schulbefragung ist auf der Homepage des Max-PlanckInstituts kostenlos zugänglich. ${ }^{20}$

Das zweite Kernelement des Projekts waren teilnehmende Beobachtungen der alltäglichen Polizeiarbeit im Umfang von etwa je 400 Stunden in Deutschland und Frankreich sowie qualitative Interviews mit je ca. 50 Polizeibeamten. ${ }^{21}$ Bei der qualitativen Feldforschung wurden in den vier Städten insgesamt ca. 500 Interaktionen zwischen Polizeibeamten und Bürgern beobachtet. Die nachfolgenden Ergebnisse stützen sich vornehmlich auf die Daten der standardisierten Schulbefragung, die anschließend mit den qualitativen Beobachtungen verglichen werden. Für die nachfolgenden Analysen ist die Definition des Migrationshintergrundes zentral, die eng gefasst ist: Der Migrationshintergrund wird dabei als Indikator für eine ethnische Zuordnung verwendet, und beide Begriffe werden im Folgenden synonym verwendet. Beide Elternteile oder mindestens drei Großeltern müssen im Ausland geboren sein. Demzufolge besteht kein Migrationshintergrund, wenn nur ein Elternteil außerhalb Deutschlands geboren wurde. Auf der anderen Seite schließt die Definition Migranten der 3. Generation ein, deren Eltern in Deutschland als Kinder von Einwanderern geboren wurden. Die ethnische Herkunft wird über das Geburtsland der Eltern und Großeltern bestimmt, was im Einzelfall zu falschen Zuordnungen führen kann. Nach dieser Definition hatten ungefähr ein Drittel der befragten Jugendlichen in Frankreich und ca. $40 \%$ der befragten Jugendlichen in Deutschland einen Migrationshintergrund. Weitere ca. $20 \%$ der französischen und $10 \%$ der deutschen Jugendlichen wuchsen in Familien mit einem einheimischen und einem migrantischen Elternteil auf. Die größten

20 Oberwittler, D./Schwarzenbach, A./Gerstner, D., Polizei und Jugendliche in multiethnischen Gesellschaften. Ergebnisse der Schulbefragung 2011 "Lebenslagen und Risiken von Jugendlichen" in Köln und Mannheim (research in brief 47), Freiburg 2014 (abrufbar unter https://www.mpicc.de/shared/data/pdf/rib_47_oberwittler_ua.pdf).

${ }^{21}$ Hunold, D., Polizei im Revier. Polizeiliche Handlungspraxis gegenüber Jugendlichen in der multiethnischen Stadt, Berlin 2015; Hunold D./Oberwittler, D./Lukas, T., "I'd like to see your identity cards please", Negotiating authority in policeadolescent encounters: Findings from a mixed-method study of proactive police practices towards adolescents in two German cities, European Journal of Criminology 13, 2016, S. 590-609; Schwarzenbach, A., A comparative view on police forces' perceptions of (minority) youths in deprived neighbourhoods in three European countries (Master Thesis, Faculty of Law, University of Berne), 2012. 
Herkunftsländer in Deutschland waren die Türkei, Russland, Polen und südeuropäische Länder, und in Frankreich die Maghreb-Staaten Algerien, Marokko und Tunesien sowie Länder in Südeuropa und Schwarzafrika. Soweit seltenere Herkunftsländer aus statistischen Gründen in Großregionen zusammengefasst werden müssen, lassen sich diese mit Einschränkungen zwischen Deutschland und Frankreich vergleichen. So dominieren bei den arabischen Ländern in der französischen Stichprobe die Maghreb-Länder Algerien, Tunesien und Marokko, während es sich in der deutschen Stichprobe überwiegend um vorderasiatische Herkunftsländer handelt.

Eine aus sechs Items wie gebildete Skala misst die Einstellung der Jugendlichen zur Polizei. Dabei sollten die Befragten ihre persönliche Haltung zu positiven und negativen Aussagen wie zum Beispiel „Insgesamt kann man der Polizei vertrauen“ oder „Die Polizei behandelt Ausländer schlechter als Deutsche“ (bzw. Franzosen) angeben. Der Wertebereich dieser Skala reicht von 1 bis 4, wobei Werte von 1,0 bis 2,5 eine negative und Werte von 2,5 bis 4 eine positive Einstellung markieren.

Um die Häufigkeit verdachtsunabhängiger Personenkontrollen zu erheben, haben wir die Schüler gefragt, ob und wenn ja, wie oft sie in den letzten zwölf Monaten Kontakt mit der Polizei in ihrer Stadt hatten. Dazu haben wir verschiedene, häufig vorkommende Anlässe für Polizeikontakte aufgelistet und die Jugendlichen gebeten, im Einzelnen die Kontaktanlässe und -häufigkeit

anzugeben. In den deutschen Städten hatten insgesamt ca. $43 \%$ der Schüler während der letzten zwölf Monate mindestens einen Polizeikontakt (Prävalenzrate), und $25 \%$ der Schüler sogar mehrere Polizeikontakte. Dieser sehr hohe Anteil muss jedoch relativiert werden, da anzunehmen ist, dass der sogenannte Telescoping-Effekt zu einer Überschätzung gefuihrt hat. Aufgrund von Nachfragen zum letzten Polizeikontakt wissen wir, dass ein Teil der berichteten Kontakte länger als zwölf Monate zurückgelegen hat.

Zwei der insgesamt sieben Kontaktanlässe werden im Folgenden verwendet, um verdachtsunabhängige Personenkontrollen zu untersuchen: „Ich bin auf der Straße/auf einem Platz usw. angesprochen oder kontrolliert worden“ sowie ,als Verkehrsteilnehmer (z.B. auf dem Rad)“. Da Begegnungen im Zusammenhang mit dem Verdacht auf Straftaten sowie selbst-initiierte Kontakte durch andere Fragen abgedeckt wurden, kommen diese beiden Kontaktanlässe dem Konzept der verdachtsunabhängigen Personenkontrollen nahe, auch wenn anzunehmen ist, dass regelwidriges Verkehrsverhalten häufiger Anlass von Verkehrskontrollen ist. Die Ansprache oder Kontrolle durch die Polizei auf der Straße war mit $19 \%$ der befragten Schüler in Deutschland die häufigste aller Kontaktanlässe; $7 \%$ berichteten über 
eine Kontrolle als Verkehrsteilnehmer und insgesamt $23 \%$ über eine oder beide dieser genannten Kontaktarten.

\section{Ergebnisse}

Betrachtet man zunächst die allgemeine Einstellung der befragten Jugendlichen zur Polizei, so zeigen sich in den beiden französischen Städten erhebliche Unterschiede zwischen einheimischen und migrantischen Jugendlichen (Abbildung 1). Die arabisch- und afrikanisch-stämmigen Jugendlichen sehen die Polizei insgesamt eher negativ, was an den Werten von 2,3 bzw. 2,4 - also noch unterhalb des neutralen Mittelpunktes von 2,5 - erkennbar ist. Dort liegen die meisten übrigen Minderheitengruppen. Lediglich der Wert der einheimisch-französischen Jugendlichen liegt mit 2,8 zwar etwas im positiven Bereich oberhalb des neutralen Mittelpunkts, aber dennoch deutlich unterhalb des Wertes der einheimisch-deutschen Jugendlichen (3,1). Deutsche Jugendliche bewerten ,ihre“ Polizei also spürbar positiver als französische. Ein zweiter und entscheidender Unterschied zwischen beiden Ländern ist, dass die Zustimmungswerte der Jugendlichen aus ethnischen Minderheitengruppen in den deutschen Städten mit dem Wert 3,0 beinahe genauso hoch und im positiven Bereich sind wie die der einheimischdeutschen Jugendlichen. Zwar sind diese Unterschiede aufgrund der großen Fallzahlen statistisch signifikant (bis auf die arabisch-stämmigen und gemischt türkisch-/deutsch-stämmigen Jugendlichen, deren Balken in Abbildung 1 daher gemustert sind), aber substantiell sind diese Unterschiede nicht. Die Einstellung der Jugendlichen zur Polizei ist demnach in den deutschen Städten generell positiver als in den französischen, und außerdem teilen auch die migrantischen Jugendlichen in Deutschland diese eher positive Haltung, während in Frankreich eine Kluft zwischen den Jugendlichen der Mehrheitsgruppe und der verschiedenen Minderheitengruppen besteht, allen voran den arabisch-stämmigen Jugendlichen, die eine deutlich negative Meinung zur Polizei haben. 


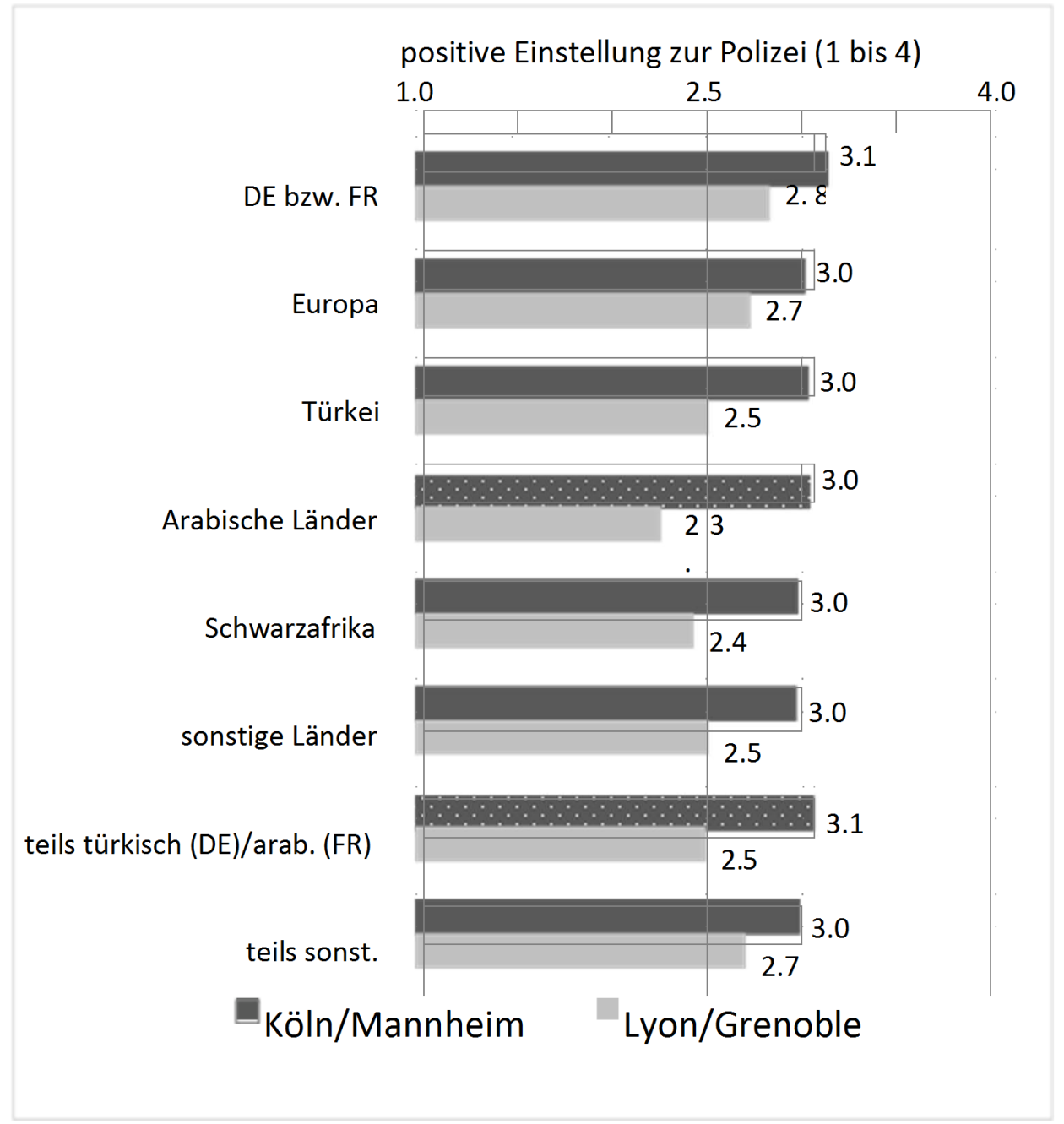

Verdachtsunabhängige Kontakte mit Polizeibeamten sind in den deutschen Städten mit ca. 23 \% häufiger als in den französischen Städten mit $18 \%$ der befragten Jugendlichen. Jungen sind in beiden Ländern ungefähr doppelt so häufig betroffen wie Mädchen. Insgesamt handelt es sich demnach um eine Alltagserfahrung vieler vor allem männlicher Jugendlicher. Viele Jugendliche werden aber im Laufe eines Jahres nicht nur einmal, sondern mehrfach angesprochen. Hier werden erste Unterschiede zwischen den beiden Ländern deutlich: Die Tabellen $1 a$ und $1 b$ enthalten die Prävalenzen und Häufigkeiten der verdachtsunabhängigen Kontakte - nur für männliche Jugendliche - nach ethnischen Herkunftsgruppen. Obwohl die französische Polizei insgesamt weniger Jugendliche kontrolliert als die deutsche, führt sie bei denselben Jugendlichen häufigere Kontrollen durch. In den deutschen Städten berichten ca. $5 \%$ aller 
Jungen, dass sie mehr als fünfmal verdachtsunabhängig Kontakt mit der Polizei hatten, in den französischen Städten sind es $8 \%$.

Tabelle la Häufigkeit verdachtsunabhängiger Personenkontrollen von männlichen Jugendlichen im letzten Jahr nach ethnischer Herkunft (Lyon und Grenoble)

\begin{tabular}{|c|c|c|c|c|c|}
\hline \multirow{2}{*}{$\begin{array}{l}\text { Herkunftsländer } \\
\text { Frankreich (kein Migr.) }\end{array}$} & \multirow{2}{*}{$\begin{array}{l}\mathrm{N}= \\
3.506\end{array}$} & & 1 bis $2^{\mathbf{n a c}}$ & $\stackrel{\text { gkeit }}{>}$ & \\
\hline & & 20.0 & 11.8 & 3.6 & 4.7 \\
\hline Europa & 386 & 26.6 & 13.7 & 5.0 & 7.8 \\
\hline Türkei & 125 & 29.1 & 7.7 & 10.3 & 11.1 \\
\hline Arabische Länder & 925 & 38.6 & 13.5 & 8.1 & 17.1 \\
\hline Schwarzafrika & 229 & 28.8 & 18.1 & 3.4 & 7.3 \\
\hline sonstige Länder & 370 & 25.9 & 11.6 & 3.8 & 10.5 \\
\hline gemischt frz.-arab. & 464 & 30.4 & 14.5 & 5.6 & 10.3 \\
\hline gemischt frz.-sonst. & 784 & 25.6 & 12.5 & 5.0 & 8.1 \\
\hline Total & 6.789 & 25.0 & 12.5 & 4.7 & 7.8 \\
\hline
\end{tabular}

Schubefragung Lyon und Grenoble, nur Jungen 
Tabelle $1 b \quad$ Häufigkeit verdachtsunabhängiger Personenkontrollen von männlichen Jugendlichen im letzten Jahr nach ethnischer Herkunft (Köln und Mannheim)

\begin{tabular}{l|r|r|rrr}
\multirow{2}{*}{ Herkunftsländer } & \multicolumn{2}{|c|}{ Prävalenz } & \multicolumn{4}{|c}{ nach Häufigkeit } \\
& & & \multicolumn{1}{|c}{ 1 bis 2 3 bis 5 } & > 5 mal \\
\hline Deutschland (kein Migr.) & 1.631 & 29.7 & 19.5 & 6.1 & 4.2 \\
Europa & 434 & 25.8 & 14.4 & 7.0 & 4.4 \\
Türkei & 714 & 30.2 & 19.0 & 5.8 & 5.4 \\
Arabische Länder & 123 & 29.3 & 15.5 & 7.3 & 6.5 \\
Schwarzafrika & 31 & 36.7 & 20.0 & 16.7 & 0.0 \\
sonstige Länder & 149 & 31.5 & 18.2 & 6.3 & 7.0 \\
gemischt dt.-türkisch & 54 & 33.3 & 13.0 & 3.7 & 16.7 \\
gemischt dt.-sonst. & 363 & 34.4 & $\underline{22.5}$ & 8.1 & 3.9 \\
\hline Total & 3.499 & 30.0 & 18.8 & 6.4 & 4.8
\end{tabular}

Schulbefragung Köln und Mannheim, nur Jungen

Die in den Tabellen $1 a$ und $1 b$ aufbereiteten Ergebnisse deuten für die französischen Städte auf eine Ungleichbehandlung von männlichen Jugendlichen nach ethnischer Herkunft hin. Die größte Minderheitengruppe der arabisch-stämmigen männlichen Jugendlichen hat eine fast zweifach erhöhte Wahrscheinlichkeit gegenüber einheimisch-französischen Jugendlichen, überhaupt kontrolliert zu werden (38\% vs. $20 \%$ ), und eine mehr als dreifach erhöhte Wahrscheinlichkeit, sehr oft kontrolliert zu werden (17\% vs. $5 \%$ ). Auch andere Minderheitengruppen zeigen höhere Kontrollraten als französische Jugendliche ohne Migrationshintergrund. Demgegenüber unterscheiden sich in Köln und Mannheim die entsprechenden Zahlen für männliche Jugendliche aus unterschiedlichen Herkunftsgruppen nicht deutlich voneinander, weder was die Prävalenz noch was die Häufigkeit von Kontrollen betrifft. Insbesondere die größte Minderheitengruppe der türkisch-stämmigen Jugendlichen wird nicht häufiger kontrolliert als die Gruppe der einheimisch-deutschen Jugendlichen.

Der sehr hohe Wert der gemischt deutsch-türkischen Jugendlichen ebenso wie der sehr niedrige der afrikanischen Jugendlichen muss aufgrund geringer Fallzahlen vorsichtig interpretiert werden.

Der diskriminierende Charakter des Ethnic Profiling liegt darin begründet, dass nur die äußere Erscheinung und nicht auch das Verhalten oder die Situation als Entscheidungskriterium für eine verdachtsunabhängige Personenkontrolle dient. Aber, so lautet ein Gegenargument, falls sich Jugendliche aus bestimmten ethnischen Minderheitengruppen in ihrem Verhalten von einheimischen 
Jugendlichen unterscheiden, falls sie z.B. häufiger und zu anderen Uhrzeiten an bestimmten öffentlichen Orten in Erscheinung treten, oder falls sie sich delinquenter verhalten, dann könnte ihre höhere Betroffenheit von Polizeikontrollen durch ihr Verhalten und nicht durch ihre ethnische Zugehörigkeit alleine erklärbar sein und wäre demzufolge auch nicht diskriminierend. Diese Hypothese kann empirisch getestet werden, indem entsprechende Indikatoren des Verhaltens der Jugendlichen in ein multivariates Erklärungsmodell der Wahrscheinlichkeit von Polizeikontakten aufgenommen werden. Wenn diese Prädiktoren dazu führen, dass die ethnische Zuordnung ihre Signifikanz verliert, wäre diese Hypothese bestätigt; andernfalls würde sich der Eindruck des diskriminierenden Verhaltens der Polizei erhärten. Da die Zielvariable, die Anzahl der Poilizeikontakte im letzten Jahr, eine schief verteilte Zählvariable ist, wird dieses Modell als nichtlineare Regressionsanalyse mit negativer Binomialverteilung gerechnet.

Abbildung $2 a$ Häufigkeit verdachtsunabhängiger Personenkontrollen von männlichen Jugendlichen im letzten Jahr nach ethnischer Herkunft - Modellvorhersage unter Kontrolle von Verhaltensindikatoren (Lyon und Grenoble)

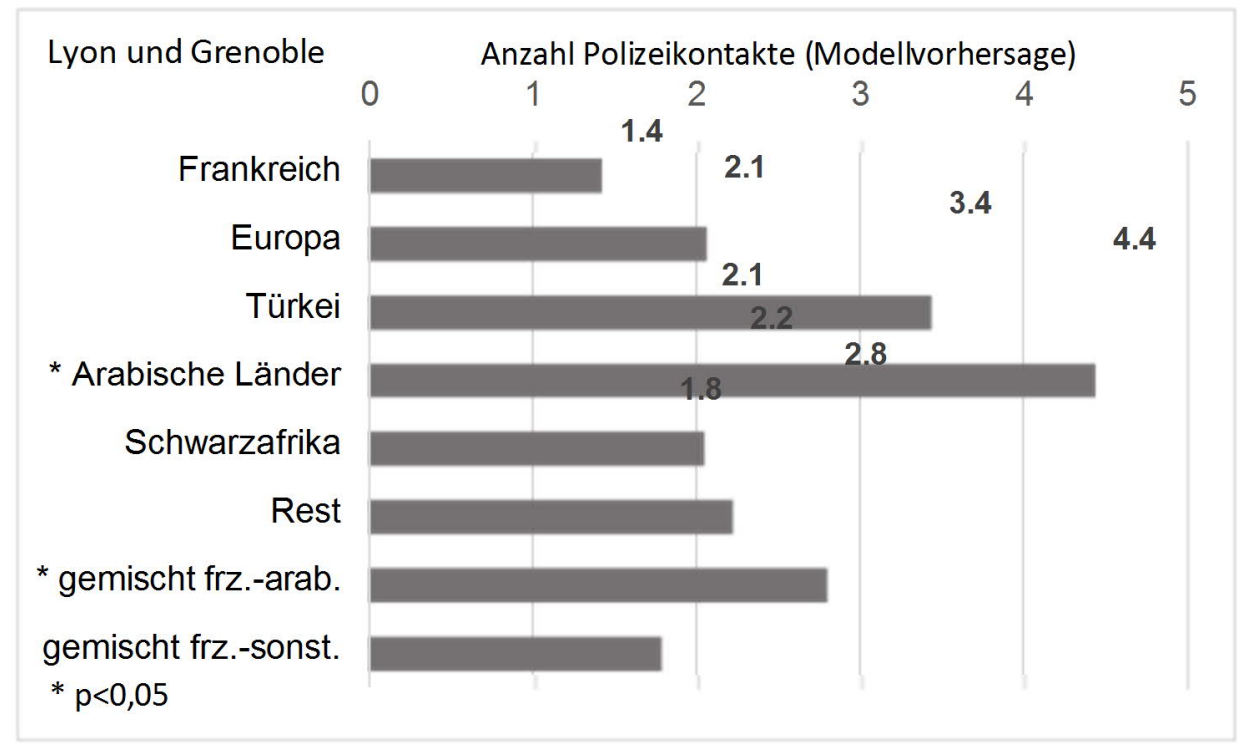




\section{Abbildung $2 b$ Häufigkeit verdachtsunabhängiger Personenkontrollen von männlichen Jugendlichen im letzten Jahr nach ethnischer Herkunft - Modellvorhersage unter Kontrolle von Verhaltensindikatoren}

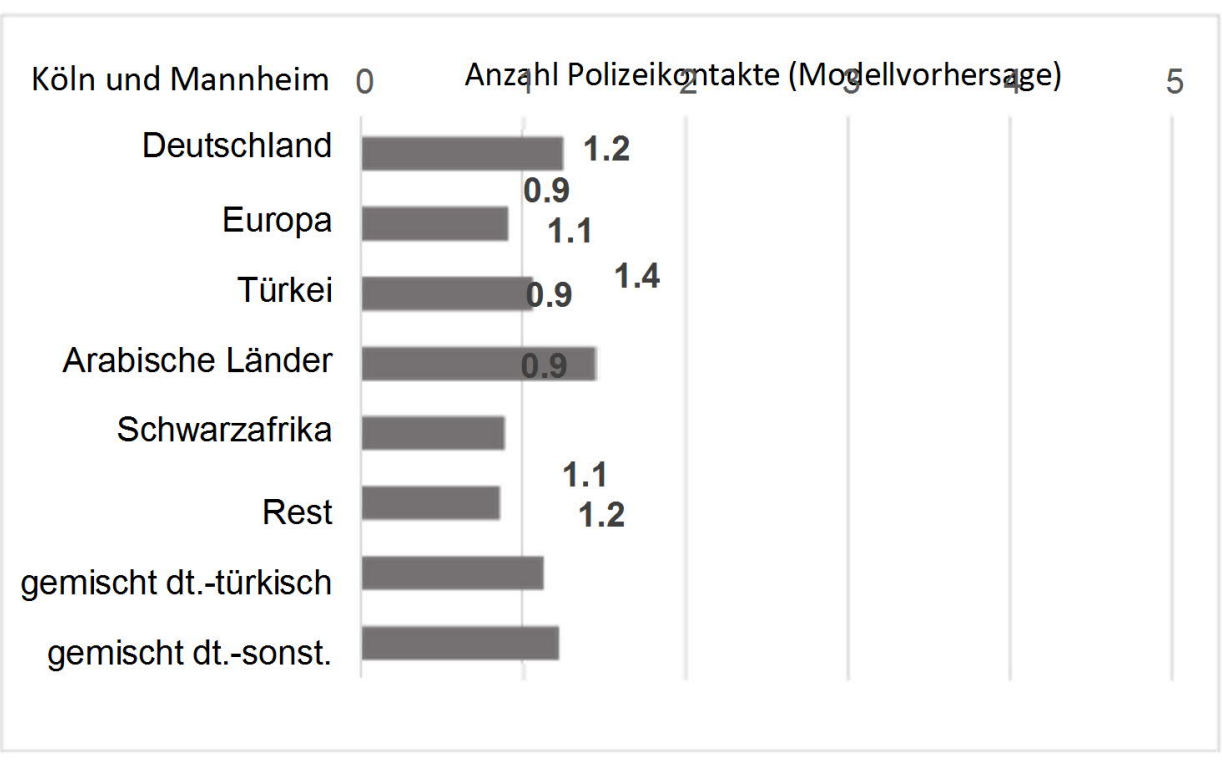

Die im multivariaten Modell unter Kontrolle aller übrigen Prädiktoren vorhergesagte Anzahl der Polizeikontakte von männlichen Jugendlichen der unterschiedlichen ethnischen Gruppen ist in den Abbildungen $2 a$ und $2 b$ graphisch dargestellt. In diesem Modell wird berücksichtigt, wie häufig sich Jugendliche nachmittags oder abends mit ihren Freunden treffen, wie häufig sie sich in ihrer Freizeit auf Straßen, Plätzen und anderen öffentlichen Räumen aufhalten, wie häufig sie betrunken waren, und wie häufig sie Straftaten wie Vandalismus, Diebstahl oder Körperverletzung begangen haben. Darüber hinaus kontrolliert das Modell auch für den Bildungsstatus und die Arbeitslosigkeit der Eltern. Alle Indikatoren eines riskanten und delinquenten Lebensstils haben in beiden Ländern einen starken und sehr signifikanten Einfluss auf die Wahrscheinlichkeit von Polizeikontrollen. Dies zeigt an, dass die Polizei bei der Anwendung verdachtsunabhängiger Kontrollen häufig „die Richtigen“ im Sinne ihres Gefahrenverständnisses trifft.

Was die Bedeutung der ethnischen Herkunft nach Kontrolle dieser Verhaltensdimension betrifft, zeigen sich in Abbildung $2 b$ für die deutschen Städte wie bereits in der deskriptiven Analyse keine nennenswerten Unterschiede zwischen den einheimisch-deutschen und migrantischen Jugendlichen. Dagegen besteht in den französischen Städten die höhere Wahrscheinlichkeit verdachtsunabhängiger Kontrollen für die männlichen Jugendlichen arabischer Herkunft auch im multivariaten Modell fort (Abbildung 2a). Das Modell berechnet für sie durchschnittlich 4,4 Polizeikontakte im Jahr gegenüber nur 1,4 Kontakten für einheimisch-französische Jugendliche. Dieser Unterschied ist statistisch sehr signifikant. Auch Jugendliche aus gemischt französisch-arabischen Familien werden signifikant häufiger 
kontrolliert (vorhergesagt 2,8 Kontrollen). Demnach haben arabisch-stämmmige männliche Jugendliche eine dreifach, und gemischt französisch-arabische männliche Jugendliche eine zweifach höhere Wahrscheinlichkeit verdachtsunabhängiger Kontrollen. Das entsprechende Modell für weibliche Jugendliche zeigt eine zweifach höhere Wahrscheinlichkeit für beide Gruppen an. Auch die Jugendlichen aus anderen ethnischen Minderheiten werden laut den Modellergebnissen häufiger kontrolliert als einheimisch-französische Jugendliche, jedoch sind diese Unterschiede weniger ausgeprägt oder aufgrund niedriger Fallzahlen nicht

signifikant. Der Unterschied zwischen den türkisch-stämmigen und einheimisch-französischen Jugendlichen verfehlt die Signifikanz nur knapp.

Aber nicht nur im Hinblick auf die Häufigkeit, sondern auch im Hinblick auf die Art und Weise, wie Personenkontrollen durchgeführt werden, unterscheiden sich die Erfahrungen der Jugendlichen unterschiedlicher ethnischer Herkunft in Frankreich. Hierzu können die Antworten der befragten Jugendlichen zu ihren Erfahrungen und ihrem eigenen Verhalten während des letzten Polizeikontakts genutzt werden. Da die Fallzahl für diese Auswertungen deutlich kleiner ist, werden in den Tabellen $2 a$ und $2 b$ wieder Jungen und Mädchen gemeinsam betrachtet. Circa $60 \%$ der einheimisch-französischen und ca. $70 \%$ der meisten anderen Jugendlichen berichten, dass ihre Identität überprüft wurde. Während dieser Unterschied nicht sehr ausgeprägt ist, behandelt die französische Polizei die Jugendlichen jedoch deutlich ungleicher, wenn es um die Kontrolle von Taschen geht, etwa um Rauschgift zu finden: $50 \%$ der einheimischen, aber $67 \%$ der arabisch-stämmigen und sogar $80 \%$ der türkisch-stämmigen Jugendlichen in Lyon und Grenoble berichten, dass der Inhalt ihrer Taschen von der Polizei kontrolliert wurde (Tabelle 2a). Außerdem geben sehr viele Jugendliche in den französischen Städten an, dass die Polizeibeamten bei dieser Personenkontrolle gewalttätig geworden seien, und wiederum ist der Unterschied zwischen den einheimisch-französischen Jugendlichen (12,5\%) und den arabisch- und türkisch-stämmigen Jugendlichen (je $37 \%$ ) sehr groß. Entsprechendes gilt für den Anteil der Jugendlichen, die berichten, sie hätten sich gegen die Polizeibeamten gewehrt. 
Tabelle $2 a \quad$ Verhalten bei der letzten verdachtsunabhängigen Kontrolle (\% „trifft zu“)(Lyon und Grenoble)

\begin{tabular}{l|c|c|c|c} 
Herkunftsländer & $\begin{array}{r}\text { Taschen- } \\
\text { kontrolle }\end{array}$ & $\begin{array}{c}\text { Identitäts- } \\
\text { kontrolle }\end{array}$ & $\begin{array}{c}\text { "Polizei wurde } \\
\text { gewalttätig" }\end{array}$ & $\begin{array}{c}\text { "habe mich } \\
\text { gewehrt" }\end{array}$ \\
\hline Frankreich & 50.1 & 58.0 & 12.5 & 15.7 \\
Europa & 65.3 & 70.3 & 23.5 & 21.5 \\
Türkei & 79.5 & 71.8 & 37.5 & 27.8 \\
Arabische Länder & 67.4 & 67.4 & 37.0 & 32.6 \\
Schwarzafrika & 63.9 & 61.1 & 22.6 & 22.1 \\
sonst. Länder & 66.9 & 66.1 & 28.4 & 31.6 \\
gemischt frz.-arab. & 59.8 & 58.7 & 24.9 & 30.0 \\
gemischt frz.-sonst. & 55.7 & 57.5 & 19.8 & 22.1 \\
\hline Total & 57.9 & 61.3 & 21.4 & 22.7
\end{tabular}

Schulbefragung Lyon und Grenoble, Jungen und Mädchen

Tabelle $2 b \quad$ Verhalten bei der letzten verdachtsunabhängigen Kontrolle (\% „trifft zu“) (Köln und Mannheim)

\begin{tabular}{l|c|c|c|c} 
Herkunftsländer & $\begin{array}{c}\text { Taschen- } \\
\text { kontrolle }\end{array}$ & $\begin{array}{c}\text { Identitäts- } \\
\text { kontrolle }\end{array}$ & $\begin{array}{c}\text { "Polizei } \\
\text { wurde } \\
\text { gewalttätig" }\end{array}$ & $\begin{array}{c}\text { "habe mich } \\
\text { gewehrt" }\end{array}$ \\
\hline Deutschland & 36.7 & 77.9 & 3.9 & 5.0 \\
Europa & 50.0 & 75.0 & 6.6 & 5.1 \\
Türkei & 48.7 & 76.3 & 5.6 & 4.4 \\
Arabische Länder & 53.3 & 80.0 & 5.3 & 5.6 \\
Schwarzafrika* & $\cdot$ & $\cdot$ &. & $\cdot$ \\
sonst. Länder & 52.6 & 73.7 & 15.0 & 4.8 \\
gemischt dt.-türkisch* & $\cdot$ & $\cdot$ & $\cdot$ & $\cdot$ \\
gemischt dt.-sonst. & 47.5 & $\underline{85.3}$ & $\underline{6.5}$ & 11.5 \\
\hline Total & 43.2 & 77.7 & 5.1 & 6.2
\end{tabular}

Schulbefragung Köln und Mannheim, Jungen und Mädchen

* Fallzahl nicht ausreichend

Diese Ergebnisse deuten auf ein sehr konfliktreiches und aggressives Verhältnis zwischen den migrantischen Jugendlichen und der Polizei in den französischen Städten hin, zumal wenn man diese Ergebnisse mit denen aus den deutschen Untersuchungsstädten vergleicht (Tabelle $2 b$ ). Hier bestehen hinsichtlich der Häufigkeit von Identitätskontrollen, die insgesamt häufiger als in Frankreich sind, keine 
Unterschiede zwischen den ethnischen Gruppen. Taschenkontrollen werden auch in den deutschen Städten häufiger bei migrantischen Jugendlichen (ca. $50 \%$ ) als bei einheimisch-deutschen Jugendlichen (37 \%) durchgeführt, was auf einen stärkeren Verdacht der Polizeibeamten gegenüber migrantischen Jugendlichen hinweist. Jedoch zeigen die weiteren Ergebnisse, dass die Kontrollen in Köln und Mannheim deutlich entspannter ablaufen und viel seltener von Aggressionen geprägt sind als die in den französischen Städten. Nur eine kleine Minderheit von $5 \%$ bzw. $6 \%$ der befragten Jugendlichen berichten über Gewalt durch Polizeibeamte bzw. ihren Widerstand gegen sie (gegenüber $21 \%$ bzw. $23 \%$ in den französischen Städten). Und noch deutlicher wird der Kontrast zwischen Frankreich und Deutschland, wenn man feststellt, dass in deutschen Städten auch bei diesen Fragen keine oder sehr geringe Unterschiede zwischen einheimischen und migrantischen Jugendlichen bestehen (Tabelle 2b).

Die Ergebnisse der Schulbefragung lassen sich so zusammenfassen: Während sich die Polizei in den deutschen Städten nicht diskriminierend gegenüber migrantischen Jugendlichen verhält (allerdings bei ihnen häufiger Taschenkontrollen durchführt) und das wechselseitige Verhältnis zwischen Polizei und Jugendlichen bei der großen Mehrheit der verdachtsunabhängigen Kontrollen entspannt ist, gilt für die französische Polizei das Gegenteil. In den französischen Städten praktiziert die Polizei bei verdachtsunabhängigen Personenkontrollen Ethnic Profiling zulasten arabisch-stämmiger Jugendlicher, und mehr als ein Drittel dieser Interaktionen mit arabischen Jugendlichen verlaufen in einem aggressiven Klima. Damit ist keine einseitige Schuldzuweisung an die Polizei verbunden, da Konflikte und Aggressionen grundsätzlich von beiden Interaktionspartnern ausgehen können.

Dass die Polizei in den französischen Städten migrantische Jugendliche häufiger kontrolliert und das Verhältnis zu den Jugendlichen dabei angespannter ist als in den deutschen Städten, wird

auch bei einer vergleichenden Auswertung der teilnehmenden Beobachtungen deutlich. ${ }^{22}$ Die Polizei in Grenoble und Lyon verfolgt eine stark auf Kriminalitätsbekämpfung - insbesondere Drogenhandel fokussierte Taktik, bei der schon geringe Verdachtsmomente für Kontrollen ausreichen. Zudem rechtfertigen einige Polizisten die bevorzugte Kontrolle arabisch-stämmiger Jugendlicher offen mit der generalisierten Annahme einer höheren Trefferquote bei dieser Zielgruppe, auch wenn sich dies in der Praxis kaum bewahrheitet. Zum anderen dienen Personenkontrollen auch als Machtdemonstration gegenüber Jugendlichen, die ihre Ablehnung oder Feindschaft gegenüber der Polizei auf der Straße z.B. durch ironische Bemerkungen offen zeigen. 
Dabei ist die Reflexion französischer Polizisten über die antagonistischen Reaktionen von Jugendlichen, die häufigen Kontrollen unterworfen werden, nur rudimentär entwickelt. Zwar führen sie die Kontrollen in der Regel formal korrekt durch, können aber angesichts des bereits bestehenden Konfliktpotenzials eine aggressive Dynamik der Interaktionen oft nicht vermeiden und verfügen nicht über wirkungsvolle Deeskalationsstrategien.

Für die deutschen Polizisten stehen Personenkontrollen von Jugendlichen dagegen überwiegend in einem Kontext, der sich deutlich von dem eben skizzierten französischen unterscheidet. Dies gilt insbesondere, aber nicht nur, für die Jugendsachbearbeiter und Bezirksbeamten der Polizei, deren Aufgabe weniger die Verbrechensbekämpfung im engeren Sinne ist, sondern eher die Problemlösung und der Ordnungserhalt in einem an Konzepte des Community Policing erinnernden Sinne. Das Bewusstsein um mögliche negative Reaktionen auf polizeiliches Handeln ist bei den deutschen Polizeibeamten stärker ausgeprägt. Daher legen diese Beamten einen besonderen Wert auf den Aufbau und die Pflege von positiven Beziehungen zu den Jugendlichen in ihrem Bezirk, was ihre Arbeit im Ernstfall erleichtern soll. Sie bemühen sich gegenüber den Jugendlichen eher als ihre französischen Kollegen um einen situationsangepassten, informellen und manchmal auch „kumpelhaften“ Ton, der in der Regel bei den Jugendlichen gut ankommt. ${ }^{23}$ Bei den meisten Personenkontrollen, die im Rahmen der Feldforschung in den beiden deutschen Städten beobachtet wurden, verliefen die Interaktionen zwischen Polizeibeamten und Jugendlichen entsprechend entspannt, und dies galt auch für die sozial besonders benachteiligten

\footnotetext{
${ }^{22}$ Vgl. hierzu ausführlicher de Maillard J./Hunold, D./Roché, S./Oberwittler, D., Different styles of policing: discretionary power in street controls by the public police in France and Germany, Policing and Society, 2016, S. 1-14; Hunold (Anm. 21); Hunold/Oberwittler/Lukas (Anm. 21); Lukas, T./Gauthier, J., Warum kontrolliert die Polizei (nicht)? - Unterschiede im Handlungsrepertoire deutscher und französischer Polizisten, in: Oberwittler, D./Behr, R. (Hrsg.), Polizei und Polizieren in multi-ethnischen Gesellschaften (Sonderheft Soziale Probleme), Pfaffenweiler 2011, S. 113-117.

${ }^{23}$ Hüttermann, J., Polizeialltag und Habitus: Eine sozialökologische Fallstudie, Soziale Welt 51, 2000, S. 7-24.
} 
Wohnquartiere. Insgesamt unterstützen die Befunde der teilnehmenden Beobachtungen die Ergebnisse der standardisierten Schulbefragung.

\section{Zusammenfassung}

Es liegt nahe, eine Verbindung zwischen den unterschiedlichen polizeilichen Praktiken verdachtsunabhängiger Personenkontrollen in den deutschen und französischen Städten und dem Vertrauen der Jugendlichen in die Polizei herzustellen. Die französische Polizei bietet mit einer diskriminierenden Anwendung von Personenkontrollen zweifelsohne Anlässe für eine eher negative bis feindselige Haltung der betroffenen Jugendlichen vor allem aus der arabischen Minderheit. Die Ergebnisse der standardisierten Befragungen ebenso wie der teilnehmenden Beobachtungen zeigen ein erhebliches Konfliktpotenzial in den alltäglichen Interaktionen zwischen Jugendlichen und Poli-

zei in Lyon und Grenoble. Dabei muss in Rechnung gestellt werden, dass Aggressionen und Respektlosigkeiten natürlich auch von den Jugendlichen ausgehen können. Insgesamt fehlt in den französischen Städten oftmals die Basis für ein entspanntes Miteinander von Polizei und migrantischen Jugendlichen, das im Vergleich dazu in Köln und Mannheim der Normalfall ist. Die deutsche Polizei schafft es, ethnische Diskriminierungen weitgehend $\mathrm{zu}$ vermeiden und die Personenkontrollen ohne Eskalationen durchzuführen.

Diese Ergebnisse sprechen für die Annahmen der Theorie der prozeduralen Gerechtigkeit, nach der die Polizei sich das Vertrauen der Jugendlichen durch faires und respektvolles Handeln „verdienen“ kann. Erklärungen, warum dies in Deutschland offenbar besser gelingt als in Frankreich, ergeben sich nicht unmittelbar aus den empirischen Daten, sondern sind sowohl in organisatorischen und strategischen Unterschieden der Polizeikräfte beider Länder als auch in den weiteren gesellschaftlichen Rahmenbedingungen zu suchen. So sind die deutschen Polizeibeamten besser ausgebildet und oftmals erfahrener und älter als die französischen. Die zentralisierten französischen Polizeiorganisationen sind weniger gut als die deutschen geeignet, sich flexibel an lokalen Bedürfnissen zu orientieren. Die deutsche Polizei verfolgt traditionell einen weiter gefassten, dem Konzept des angelsächsischen Community Policing verwandten Ansatz zur Bewältigung lokaler Probleme, während die französische Polizei die 
Kriminalitätsbekämpfung im engeren Sinne als wesentlichen Kern ihrer Aufgaben sieht. Dies führt möglicherweise zu einer Geringschätzung des Wertes positiver „public relations“ zu Bevölkerungsgruppen, auf deren Akzeptanz die Polizei für eine erfolgreiche Arbeit jedoch angewiesen ist. Das Verhältnis zwischen Polizei und ethnischen Minderheiten ist jedoch nicht ausschließlich das Resultat polizeilichen Handelns, sondern wird auch durch die gesellschaftliche Integration von Jugendlichen aus ethnischen Minderheiten bzw. deren Mangel mit geprägt. ${ }^{24}$ Ungleiche Arbeitsmarktund Zukunftschancen, von denen Jugendliche aus ethnischen Minderheiten in Frankreich spätestens seit der Finanzkrise deutlich stärker betroffen sind als in Deutschland, dürften gesellschaftliche Desintegrationstendenzen antreiben, die sich auch in Spannungen zwischen Jugendlichen und den Repräsentanten des Staates manifestieren.

Prof. Dr. phil. Dietrich Oberwittler, Max-Planck-Institut für ausländisches und internationales Strafrecht, Günterstalstr.73, 79100 Freiburg i.Br., E-mail: d.oberwittler@mpicc.de

${ }^{24}$ Siehe Anm. 4. 